\title{
CONSTRUCTION OF APODISED ZONE PLATES FOR SOLAR X-RAY IMAGE FORMATION
}

\author{
J. H. DIJKSTRA, W. DE GRAAFF, and L. J. LANTWAARD \\ Space Research Laboratory, Utrecht, The Netherlands
}

A Fresnel zone plate (sometimes called a Fresnel-Soret zone plate) is an image formation device consisting of a large number of concentric circular rings which are alternatively transparent and opaque for the radiation concerned.

For radiation of a given wavelength and a given focal distance of the zone plate the radii of subsequent rings are determined by the condition that for a coherent wave front propagating parallel to the axis of the zone plate the waves passing through these rings and diffracted towards the axis arrive at the focus in phase with each other.

For first order images this leads to the following well-known expression for the radius of the $n$th circle:

$$
r_{n}^{2}=n \lambda f+\frac{1}{4} n^{2} \lambda^{2}
$$

where the last term in general can be neglected when $n \lambda$ is small compared with the focal distance $f$ (Figure 1).

A characteristic feature of the zone plate as compared with e.g. a reflecting telescope is the monochromatic character of the images. A disadvantage is the presence of the so-called zero order radiation, consisting of a superposition of shadow images of the zone plate, which may considerably reduce the contrast and definition of the image (Figure 2). Deconvolution, i.e. reconstruction of the original image from the distorted one is very difficult. A better solution seems to be the use of zone plates having a sufficiently large opaque central section. In order to avoid the distortion of the image of the sun by zero order radiation the diameter of the opaque central section has to be at least twice the diameter of the solar image. The result is a ring-shaped or apodised zone plate.

The dimensions and structure of the $z$ plate present some problems. For $500 \AA$ radiation and a focal distance of $50 \mathrm{~cm}$ the widths of the rings are of the order of $2 \mu$. In addition, the transparent zones have to be completely open, so the rings have to be supported by a system of radial struts.

These circumstances greatly enhance the problems connected with the manufacture of these zone plates. An attempt to produce a zone plate on a thin parlodion foil was unsuccessful: the foil was insufficiently rigid for the purpose.

Earlier zone plates were made with the aid of electron optical imaging (Bol Raap et al., 1969), but the field of view of the available system was too small for the larger ring-shaped zone plates. Therefore it was decided to investigate the possibilities of holographic techniques, and to make use of the zone pattern produced by the interference of two coherent spherical wave fronts. The advantages of such a method are obvious: the instrumental facilities necessary are very simple; the dimensions of the 
zone pattern are flexible; and the pattern is perfectly circular in shape and everywhere sufficiently sharp.

Initial efforts to use a two-step procedure (the interference pattern was produced on ordinary high-resolution photographic film and then printed into photoresist by means

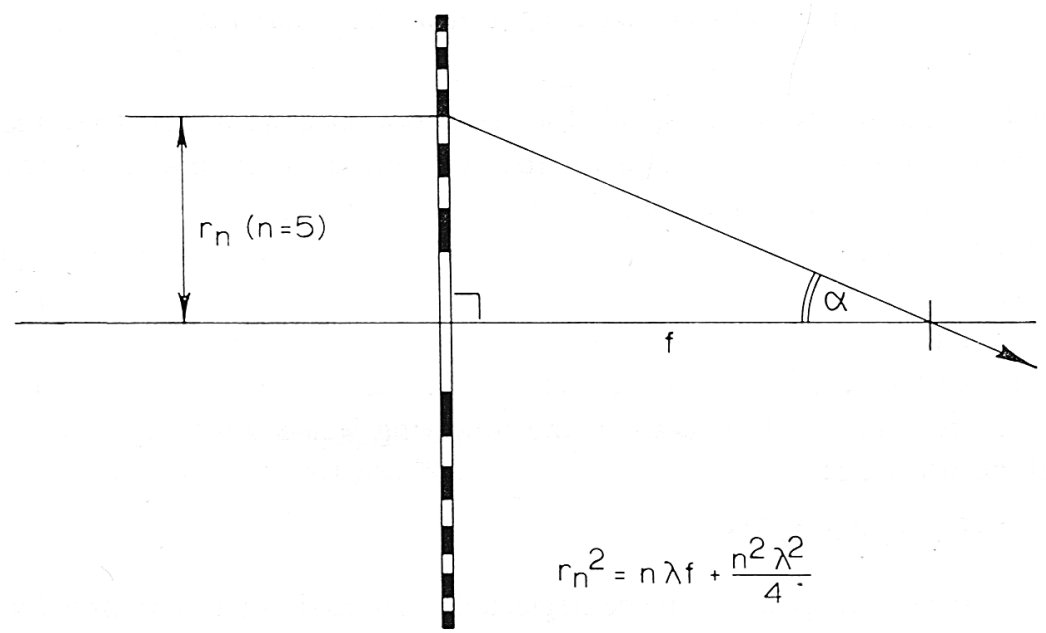

Fig. 1. Image formation conditions for a zone plate.

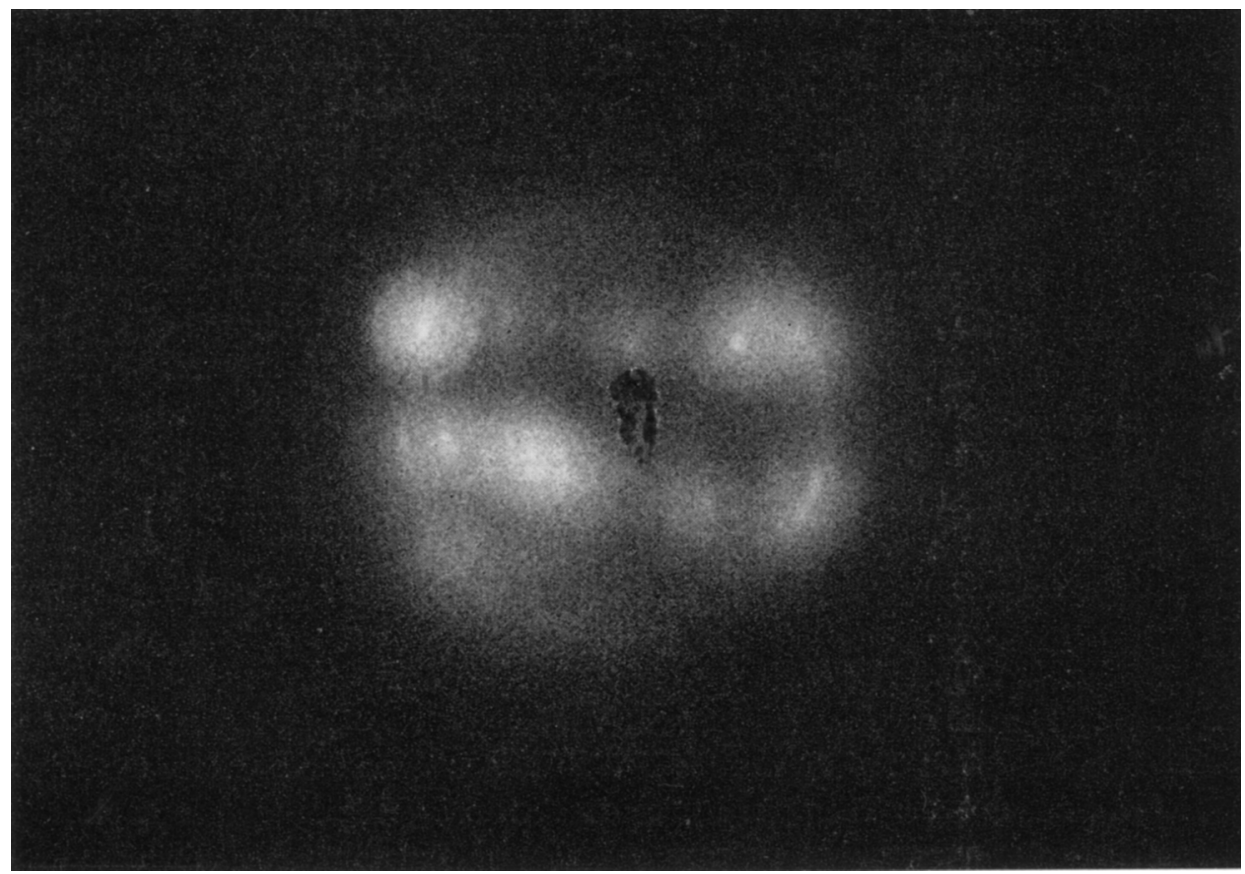

Fig. 2. Image of the sun in $51 \AA$ radiation obtained with a non-apodised zone plate camera on October 3, 1967. Original image diameter $4 \mathrm{~mm}$. 
of contact printing or imaging with a special UV lens) were unsuccessful due to exposure difficulties. Therefore another method was used involving electroplating on some suitable metal coated with the photoresist pattern and selective etching.

The $4416 \AA$ radiation of a cadmium-helium laser is split up into two beams, which are converted into spherical wave fronts by means of two microscope objectives (Figure 3). Special attention has to be given to the homogeneity of the beams in order to ensure uniform exposure across the whole area of the zone plate.

After exposure and developing, the support structure mask is reproduced on the

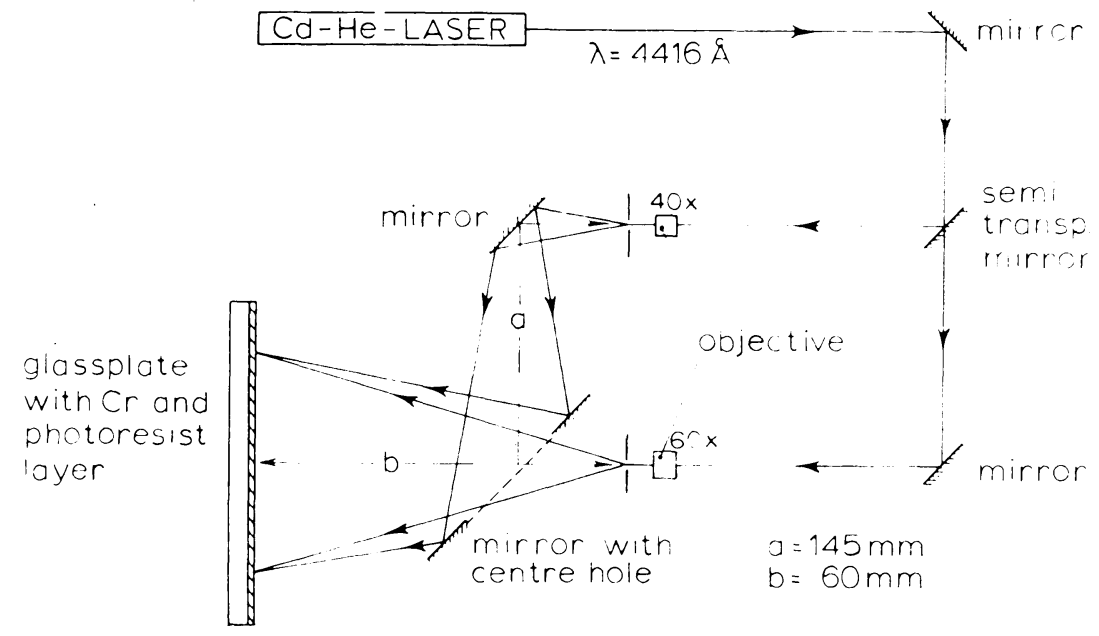

Fig. 3. Optical arrangement for the production of zone plates with a split-up laser beam.
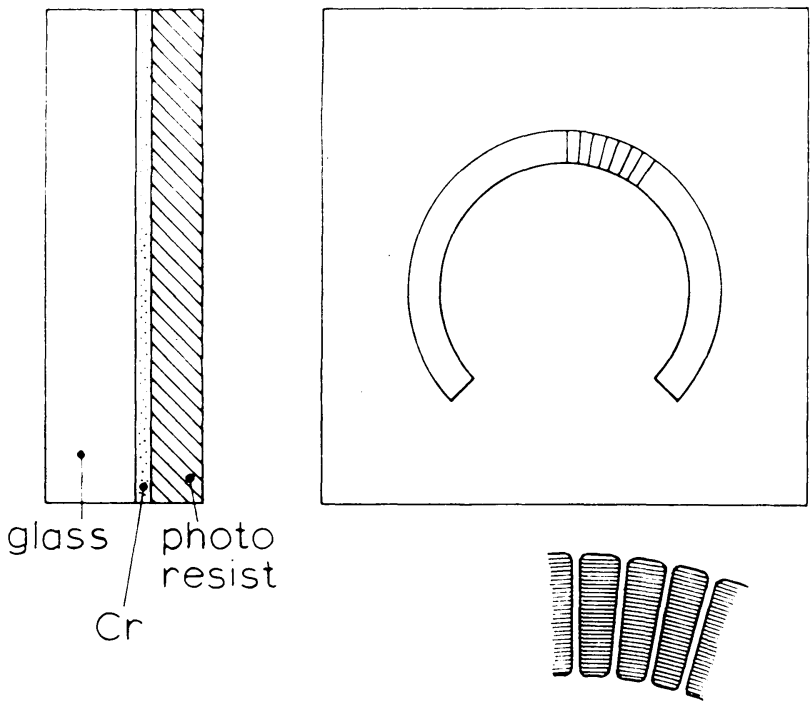

Fig. 4. First stage of the production of a zone plate. 
remaining not fully developed photoresist by means of contact printing (Figure 4). After this the zone plate itself is produced by means of nickel plating on top of the chromium layer.

Finally the zone plate structure is reinforced for mounting purposes (Figure 5). The cover paint defines the outer limit. Electroplating is continued until the required zone width is obtained, and the necessary layer thickness is reached. Then the cover paint is
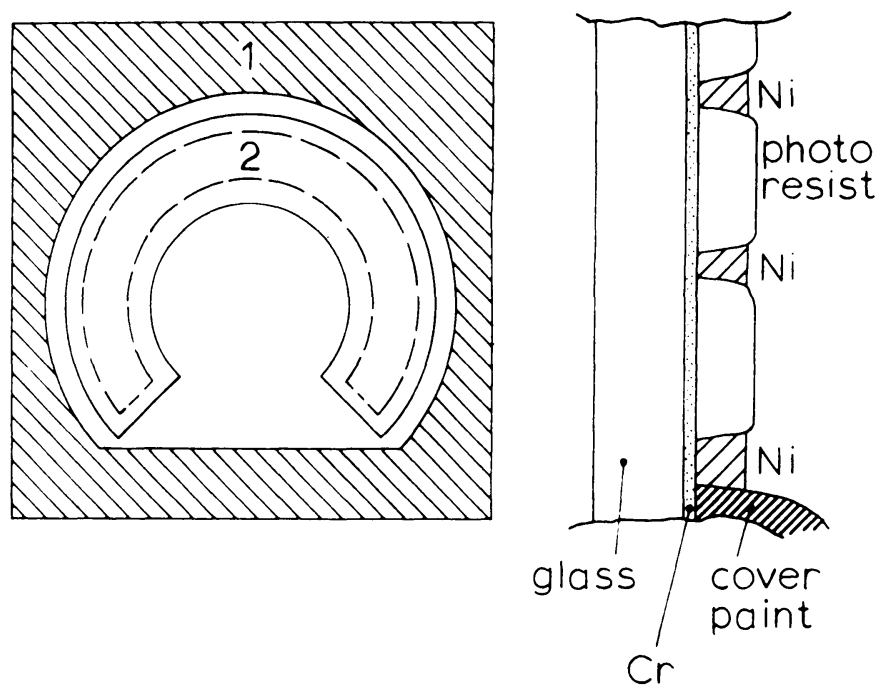

Fig. 5. Final stage of the producing of a zone plate (2) Within a reinforced holding sheet (1).

rinsed away with acetone and the nickel zone plate becomes detached from the chromium substrate.

\section{Acknowledgement}

Our thanks are due to Ir H. van de Stadt (Astronomical Institute, Utrecht) for his advice concerning the optical system and the laser.

\section{Reference}

Bol Raap, B. E., Le Poole, J. B., Dijkstra, J. H., Graaff, W. de, and Lantwaard, L. J.: 1969, in (ed.), Small Rocket Instrumentation Techniques, Amsterdam, p. 203.

\section{DISCUSSION}

J. H. Underwood: How much of the light goes into each order? Does the apodization help the problem of confusion of orders (other than zero order)?

$J$. H. Dijkstra: The average intensity in the different zone plate orders is: $25 \%$ in zeroth order and $10 \%$ in 1 and $\cdots 1$ order of incident intensity; other orders much less.

The apodization does not remove the undesirable orders other than the zeroth. 